\title{
SoC Implementation of VGA Driver using Spartan 3AN Series FPGA
}

\author{
Ramandeep Singh \\ Assistant Professor, \\ EECE Department \\ ITM University, \\ Gurgaon, India.
}

\author{
Nikhil Ahuja \\ EECE Department, \\ ITM University, \\ Gurgaon, India
}

\begin{abstract}
As the microelectronic industry grows there has been an exponential increase in the importance of FPGAs in modern digital circuit design. FPGAs not only provided an advantage of being reconfigurable but also come in extremely small packaging. In this paper we use the Xilinx Spartan 3AN series FPGA and propose its use as part of a System on Chip controller for a Video Graphics Array (VGA), that serves as one of the most popular display units today.
\end{abstract}

\section{General Terms}

VLSI Design, FPGA

\section{Keywords}

FPGA, VGA, SOC, Xilinx Spartan 3AN, RTL View.

\section{INTRODUCTION}

The System involves the use of the Spartan 3AN Series FPGA board to drive the VGA monitor. Included in the system is also a means of providing external inputs to the FPGA via a switch panel and a series of LEDs which find use as status or error indicators. VHDL is used as the Hardware Definition Language which is synthesized and proper hardware is implemented using Xilinx ISE $12.4^{[1,2]}$.

\section{COMPARISON BETWEEN FPGA, CPLD AND ASIC}

\begin{tabular}{|l|l|l|l|}
\hline & FPGA & CPLD & ASIC \\
\hline Performance & High & Low & High \\
\hline $\begin{array}{l}\text { Power } \\
\text { Consumption }\end{array}$ & High & Very High & Low \\
\hline $\begin{array}{l}\text { Test Development } \\
\text { complexity }\end{array}$ & Very Low & High & Low \\
\hline Density & High & Low & Very High \\
\hline Unit Cost & High & Low & High \\
\hline Timing Iterations & High & Very High & Low \\
\hline Design Effort & Low & High & Medium \\
\hline Memory & Volatile & $\begin{array}{l}\text { Non- } \\
\text { volatile }\end{array}$ & Both \\
\hline Security & Low & Very High & High \\
\hline
\end{tabular}

Table 1: Comparison between FPGA, CPLD and ASIC

The comparison indicates the low performance of the CPLD as compared to the FPGA and the ASIC so it was not used. The need was to choose between the FPGA and the ASIC and the FPGA was selected due to its faster time to market, simpler design cycle and the option of field reprogramability.

\section{COMPARISON BETWEEN THE DIFFERENT MEMBERS OF THE SPARTAN-3 FAMILY}

\begin{tabular}{|l|c|c|c|c|c|}
\hline Device & XC3S200 & XC3S400 & XC3S1000 & XC3S1500 & XC3S2000 \\
\hline $\begin{array}{l}\text { System } \\
\text { Gates }\end{array}$ & $200 \mathrm{~K}$ & $400 \mathrm{~K}$ & $1000 \mathrm{~K}$ & $1500 \mathrm{~K}$ & $2000 \mathrm{~K}$ \\
\hline Logic Cells & 4,320 & 8,064 & 17,280 & 29,952 & 46,080 \\
\hline $\begin{array}{l}\text { Block RAM } \\
\text { Bits }\end{array}$ & $216 \mathrm{~K}$ & $288 \mathrm{~K}$ & $432 \mathrm{~K}$ & $576 \mathrm{~K}$ & $720 \mathrm{~K}$ \\
\hline $\begin{array}{l}\text { Distributed } \\
\text { RAM Bits }\end{array}$ & $30 \mathrm{~K}$ & $56 \mathrm{~K}$ & $120 \mathrm{~K}$ & $208 \mathrm{~K}$ & $320 \mathrm{~K}$ \\
\hline $\begin{array}{l}\text { Max Single } \\
\text { Ended I/O }\end{array}$ & 173 & 264 & 391 & 487 & 712 \\
\hline $\begin{array}{l}\text { Max } \\
\text { Differential } \\
\text { I/O }\end{array}$ & 76 & 116 & 175 & 221 & 312 \\
\hline
\end{tabular}

Table 2: Comparison between different members of the Spartan- 3 family

The table discusses some of the basic architectural and memory specifications of the Spartan 3 family member of FPGAs. These parameters especially play an important role in helping to determine the particular FPGA required for the desired application.

Xilinx as a manufacturer makes available FPGAs with an array of configurations to facilitate users to make an informed choice.

The XC3S700AN FPGA was used as it lies in an intermediate product range which is suitable for utilization for the purpose of developing a prototype for a basic driver for the VGA graphics. High performance is not a primary requirement and the memory available with this Xilinx FPGA is adequate as per the requirement.

Xilinx provides an FPGA user development board which includes hardware blocks which may need to be interfaced with the FPGA. Out of the available blocks the VGA port, the LEDs and the push buttons are the ones that are required for the application. 


\section{BLOCK DIAGRAM}

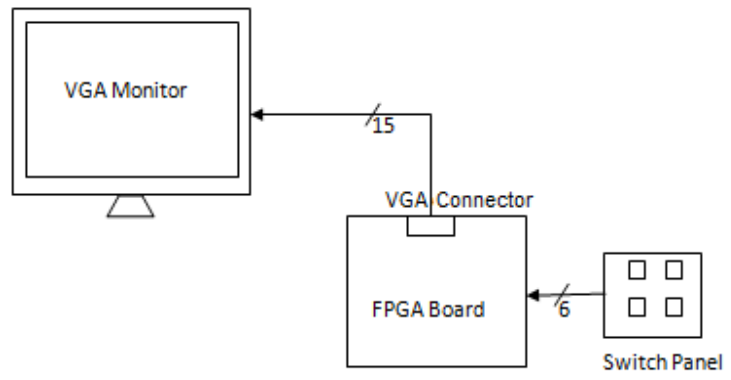

Figure 1: Block Diagram of System

VGA monitor controller, which is a logic circuit to control the VGA interface, can be easily realized by FPGA technology with a low cost and high flexibility. ${ }^{[3]}$

The block diagram shows a VGA monitor connected to the FPGA board via a standard VGA cable using a 15 pin VGA connector on the board. A switch panel consisting of four switches is connected to the FPGA board. The application will involve using the FPGA to drive the VGA screen. A variety of patterns may be displayed on the screen and the switches could be utilized to manipulate the display pattern.

\section{INTRODUCTION TO VGA}

The Video Graphics Array (VGA) is a display standard that was introduced in $1987^{[4]}$. The VGA screen that we intend to use is one which finds use in PCs today. Assuming that the VGA screen is based on the CRT technology, the following are certain important parameters that govern the display on the VGA screen.

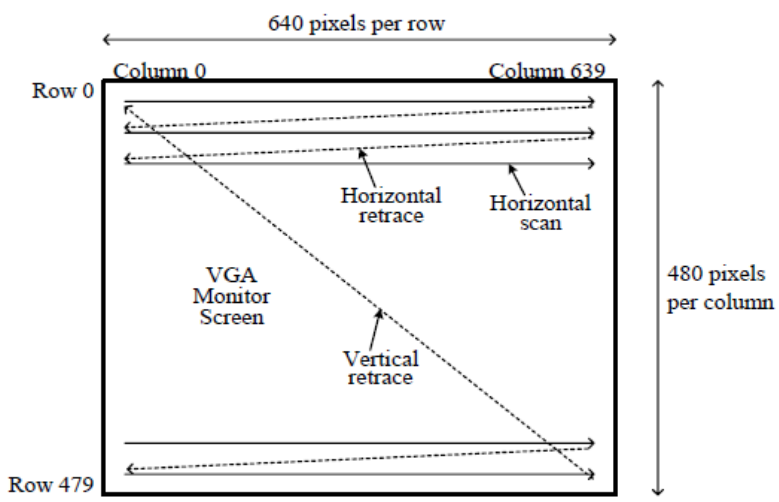

Figure 2: The VGA Monitor. The scanning starts from $(0,0)$ and moves to the right and down until reaching column 639 and row 479.

For a standard VGA Format the monitor screen contains 640 columns by 480 rows of picture elements called pixels. ${ }^{[5]}$
All pixels are scanned in raster order at a frequency called pixel frequency. The whole image will be re-drawn at a rate determined by refresh rate in order to ensure the visual quality. The pixel frequency depends on the display resolution and the refresh rate, so it is evident that if a better resolution or a higher fresh rate is desired it will require a higher pixel frequency. ${ }^{[6]}$

RGB signals are sent to appropriate pixels in rising clock pulse. The positions of the pixels are changed in each new frame, if movement of objects is desired. ${ }^{[7]} \mathrm{A}$ new frame is characterized by a rising Vertical sync pulse.

\subsection{Horizontal Sync}

The Horizontal Sync bit is used in order to indicate to the signal the beginning of a new line. The horizontal sync signal is a single short pulse which indicates the start of every line. The signal traces a line from one side to the other and a very fast retrace back to the first pixel of the next line.

\subsection{Vertical Sync}

At the end of each frame of video there is a vertical sync signal that indicates when the next frame is going to start. The vertical sync signal tells the monitor to start displaying a new image or frame. In order to do so, the monitor starts in the upper left corner with pixel $(0,0) .{ }^{[8]}$

\subsection{Refresh Rate}

This parameter specifies how fast the screen should be refreshed. For a human eye, the refresh rate must be at least 30 screens per second to make the motion appear to be continuous. To reduce flickering, the monitor usually has a much higher rate.

\section{Introduction to FPGA Development Platform}

The following section provides an insight into the basic FPGA development platform used.

\subsection{Spartan 3AN Series FPGA}

The Spartan®-3AN FPGA family combines the best attributes of a leading edge, low cost FPGA with nonvolatile technology across a broad range of densities. The Spartan-3AN FPGA family is excellent for spaceconstrained applications. ${ }^{[9]}$

Features ${ }^{[9]}$

- Integrated robust configuration memory

- Availability of up to $11 \mathrm{~K}$ non-volatile user memory

- 20 years Flash memory data retention

- Configuration watchdog timer

- Suspend mode reduces system power consumption

- Full hot-swap compliance

- Abundant, flexible logic resources

- $\quad$ Eight global clocks and eight additional clocks per each half of device

- Abundant low-skew routing 


\subsection{Connector}

The Spartan 3AN FPGA Starter Kit board includes a VGA display port via a standard high-density HD-DB15 female connector.
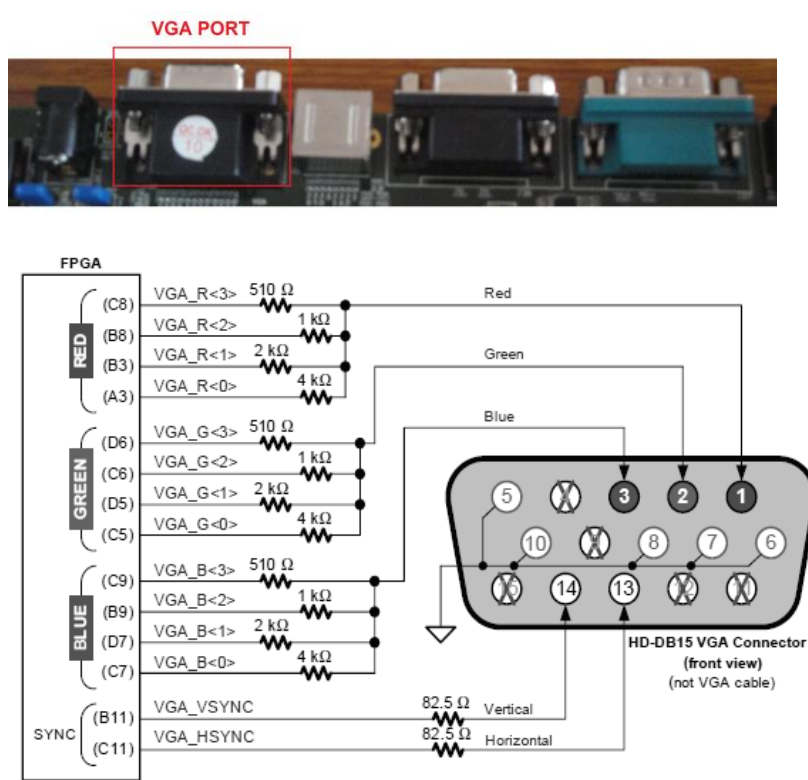

Figure 3: Standard HD-DB15 female connector, location on board and connection diagram ${ }^{[10]}$

There are three data signals for red, green and blue which control the color and two control signals horizontal synchronization and vertical synchronization signals ${ }^{[11]}$.

The FPGA is capable of directly driving the five VGA signals via resistors. The red, green, and blue signals have four outputs from the FPGA that feed a resistor-divider tree.

\subsection{Discrete LEDs}

The Spartan 3AN Starter Kit board has eight individual surface-mount LEDs located immediately above the slide switches as shown in the figure. The LEDs are labeled LED7 through LED0. ${ }^{[10]}$

LEDs can be used as indicators, for which they can be connected to the switches. These LEDs can be used to indicate errors as a result of which debugging the circuit would become convenient.

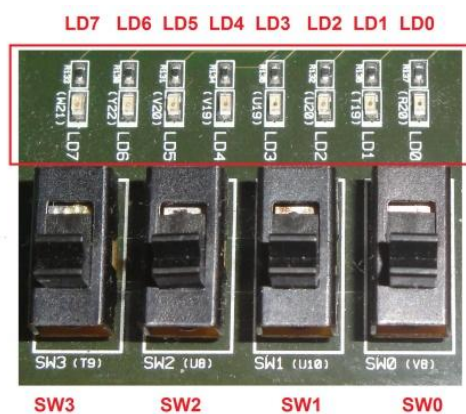

Figure 4: The eight LEDs and four switches
Each LED has one side connected to ground and the other side connected to a pin on the device via a $390 \Omega$ current limiting resistor. For lighting an individual LED, it is required to drive the associated FPGA control signal High

The UCF location constraints of the eight LEDs are mentioned below, along with the I/O pin assignment and the I/O standard used.

NET "LED $<7>$ LOC = "W21" | IOSTANDARD = LVCMOS33 $\mid$ SLEW = SLOW $\mid$ DRIVE = 8;

NET "LED<6>" LOC = "Y22" | IOSTANDARD = LVCMOS33 $\mid$ SLEW = SLOW $\mid$ DRIVE = 8;

NET "LED<5>" LOC = "V20" | IOSTANDARD = LVCMOS33 $\mid$ SLEW = SLOW $\mid$ DRIVE $=8$;

NET "LED<4>" LOC = "V19" | IOSTANDARD = LVCMOS33 $\mid$ SLEW = SLOW $\mid$ DRIVE = 8;

NET "LED $<3>"$ LOC = "U19" | IOSTANDARD = LVCMOS33 $\mid$ SLEW = SLOW $\mid$ DRIVE = 8;

NET "LED $<2>"$ LOC = "U20" | IOSTANDARD = LVCMOS33 $\mid$ SLEW = SLOW $\mid$ DRIVE $=8$;

NET "LED $<1>"$ LOC = "T19" | IOSTANDARD = LVCMOS33 $\mid$ SLEW = SLOW $\mid$ DRIVE = 8;

NET $" L E D<0>"$ LOC $=$ "R20" | IOSTANDARD = LVCMOS33 $\mid$ SLEW $=$ SLOW $\mid$ DRIVE $=8 ;{ }^{[12]}$

\subsection{Switch Panel}

The Spartan 3AN Starter Kit Board has available four momentary contact push button switches. These switches are located at the lower right corner of the board and are labeled BTN_NORTH, BTN_SOUTH, BTN_EAST and BTN_WEST as indicated in the figure.

These buttons are used to provide an input signal to the FPGA and hence this panel of switches may be used as inputs to bring variations in the graphics displayed on the VGA.

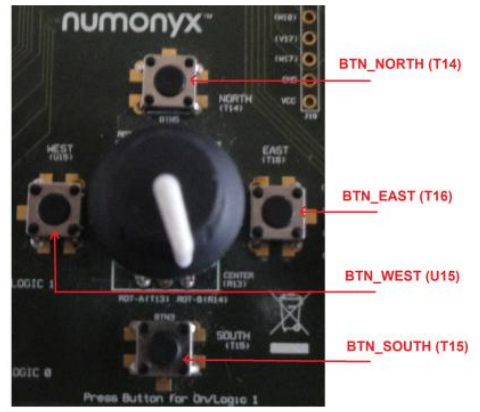

Figure 5: The four push button switches

The UCF location constraints of the four push buttons are mentioned below, along with other necessary details

NET "BTN_EAST" LOC = "T16" | IOSTANDARD = LVCMOS33 | PULLDOWN; 
NET "BTN_NORTH" LOC = "T14" | IOSTANDARD = LVCMOS33 | PULLDOWN;

NET "BTN_SOUTH" LOC = "T15" | IOSTANDARD = LVCMOS33 | PULLDOWN;

NET "BTN_WEST" LOC = "U15" | IOSTANDARD = LVCMOS33 | PULLDOWN; ${ }^{[12]}$

\section{Pattern Design in VGA}

The following section carries information regarding the methods used to display various kinds of graphics on screen.

\subsection{Analysis of shape Formation}

\subsubsection{Method of displaying a rectangle/square}

A rectangular object can be defined by its boundary in terms of pixel coordinates on the screen. The figure illustrate the pixels that need to be lit in order to obtain the desired shapes.
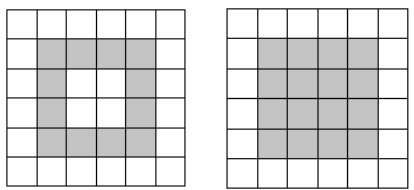

Figure 6: Bit Map of a Square and a filled Square

\subsubsection{Method of displaying a circle}

A circle will be defined in a square boundary that encloses it. The requirement for drawing a circle remains the definition of this square boundary. This boundary may be defined using the radius or the diameter of the circle as reference. Once the boundary is defined, the pixels are lit to display a circular shape. The figure illustrates a circle boundary and a filled circle drawn within an 8 -by- 8 pixel square.
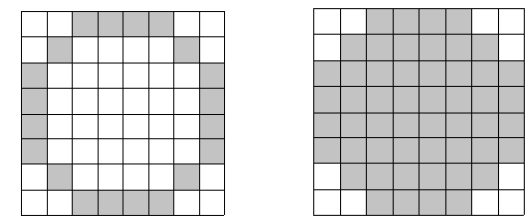

Figure 7: Bit Map of a Circle and a filled circle in an 8by-8 pixel Square

\subsubsection{Method of displaying a Text/Character ${ }^{[13]}$}

The letters of the alphabet as well as numbers and special characters can all be displayed using straight lines and curves. When looking to display text, the parameters that play an important role include the. The figure shows the letters $\mathrm{V}, \mathrm{G}$ and $\mathrm{A}$ constituting VGA each displayed in an 8-by-8 pixel square.
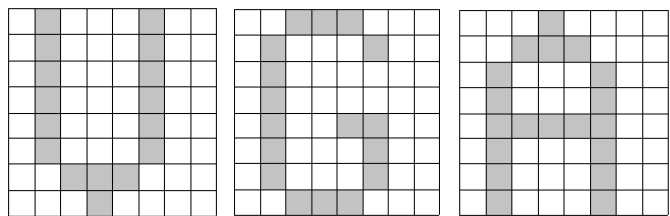

Figure 8: Bit Map of the letters $V, G$ and $A$ each displayed in an 8-by-8 pixel square

\section{GENERATED RTL VIEW}

The figure below is an indication of the RTL view for an FPGA designed to display a single horizontal line. The RTL view has been split into two sections and if interconnectivity is provided between the two blocks along the lines labeled from A to G, the complete RTL view would be available. The entire circuitry comprises various logic blocks that would combine to form the $\mathrm{SoC}$ for driving the VGA

The RTL view indicates one input i.e. clk50 in which wil come from the crystal on the board to the FPGA. There are also output signals for the red, green and blue components of the VGA input and the horizontal sync and vertical sync signals.

The various blocks that may be required to display a single horizontal line include logic blocks that are determined by the HDL code that is written for the same. In the HDL code the pixels of the line are mentioned using greater than and less than operators to indicate the starting pixel and the ending pixel.

Also included in the RTL view are basic logic gates, multiplexers and latches which enable decision making for the horizontal sync and the vertical sync signals that act as control signals for the VGA screen. 


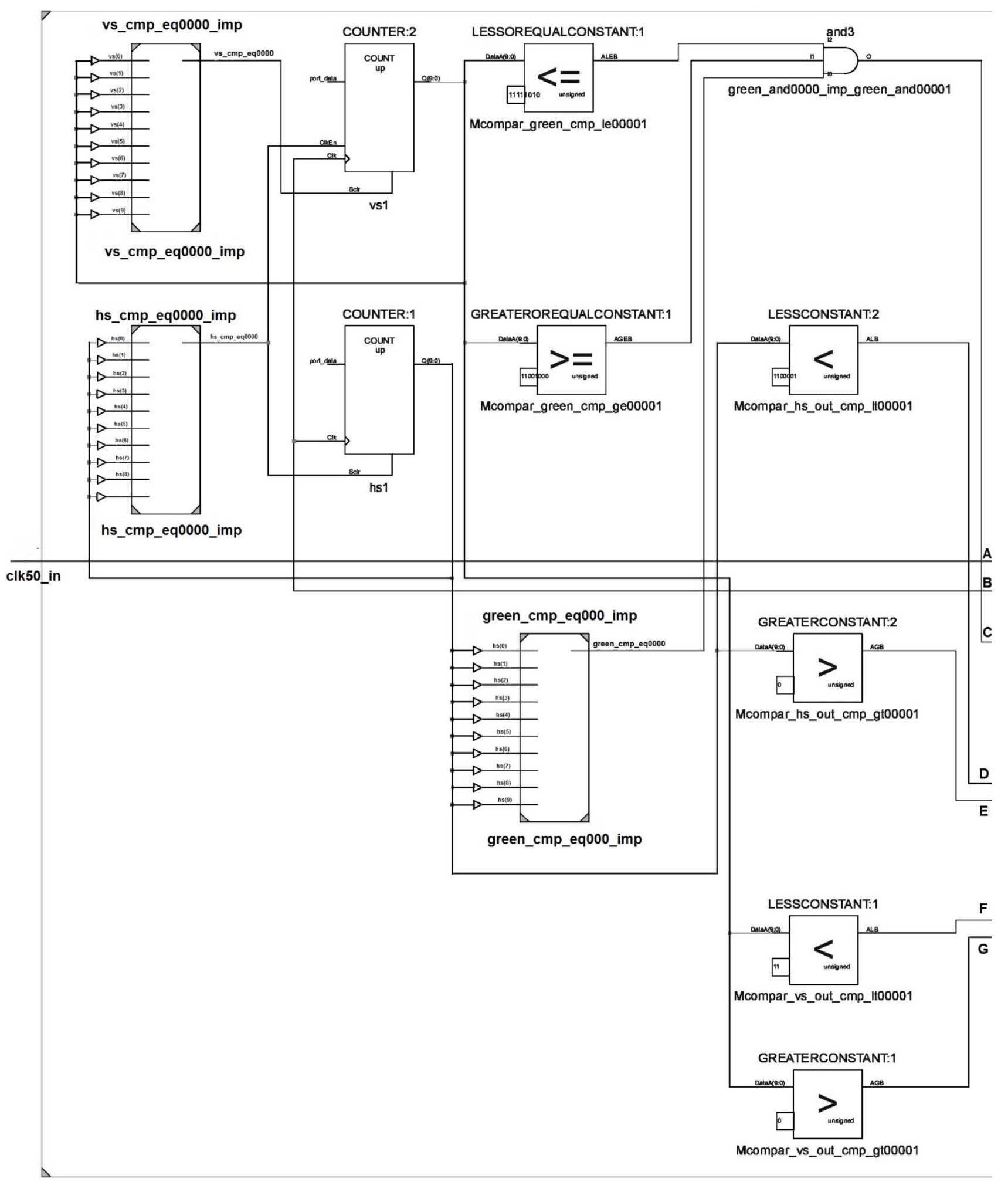

vga

Figure 9a: RTL view of SOC for displaying a single horizontal line on VGA ${ }^{[14]}$. 


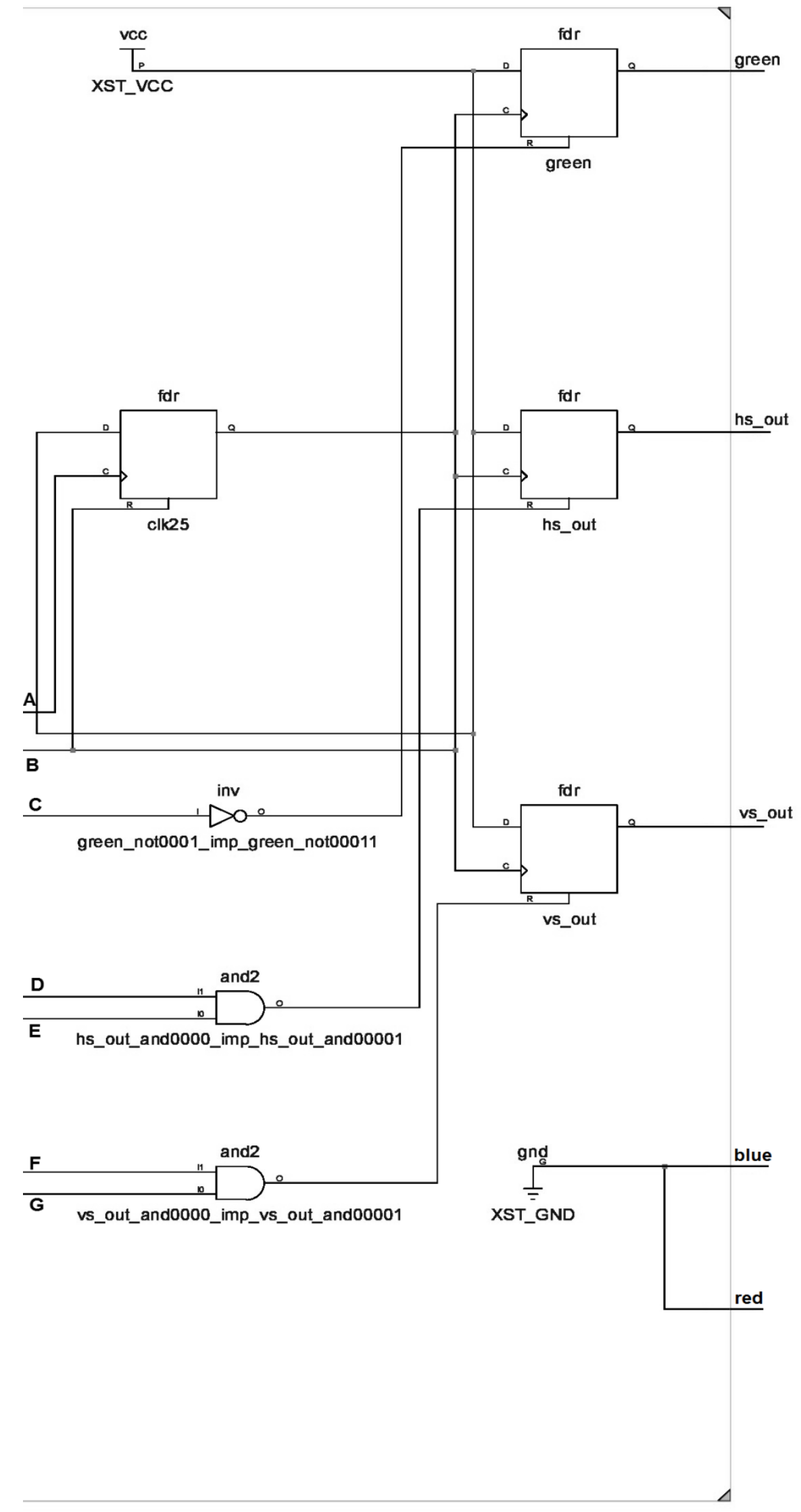

Figure 9b: RTL view of SOC for displaying a single horizontal line on VGA ${ }^{[14]}$. 


\section{RESULTS}

The following are some of the outputs that were achieved on the implementation of the system on chip using VHDL. $[1][15][16]$

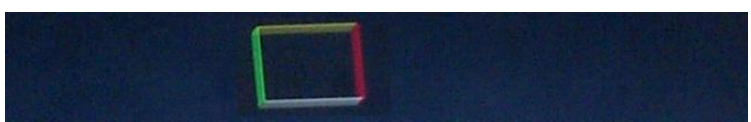

Figure 11: Display of box on VGA using the SOC

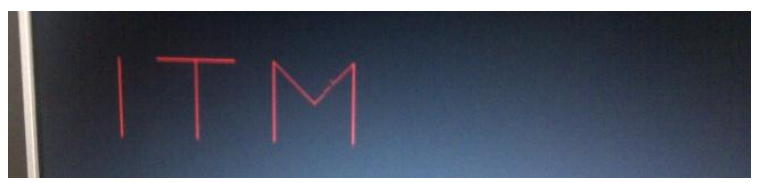

Figure 12: Display of characters on VGA using the SOC

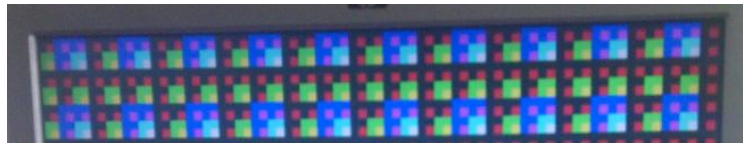

Figure 12: Display of multi-colored boxes in various sizes on VGA using the SOC

\section{ACKNOWLEDGMENTS}

Thanks to the Department of Electrical Electronics and Communication Engineering (EECE), at ITM University, Gurgaon for the use of its laboratories and resources that were made available for carrying out the above said research.

\section{CONCLUSIONS}

The system on chip for the implementation of a VGA driver on the FPGA has been designed and synthesized for various different graphics which include shapes, text and graphics in motion using Xilinx ISE Design Suite 12.4 and the Spartan-3A/3AN FPGA Starter KitlBoard.

\section{REFERENCES}

[1] IEEE Standard VHDL Reference Manual

[2] Xilinx Inc. "ISE Design Suite 12.4 Release Notes an Installation Guide", www.xilinx.com

[3] Van-Huan Tran, Xuan-Tu Tran "An Efficient Architecture Design for VGA Monitor Controller," SIS Laboratory, University of Engineering and Technology, VNU Hanoi, International Conference on Consumer Electronics, Communications and Networks (CECNet), April 2011.
[4] Programming languages: A short history for

Economists, Marc Nerlove, Department of Agricultural and Resource Economics, University of Maryland, College Park, Journal of Economic and Social Measurement Vol. 29 pp. 189-203 (2004)

[5] Building a VGA monitor controller by Enoch Hwang, Nov 2004, www.circuitcellar.com

[6] Ashish B. Pasaya, Kiritkumar R. Bhatt "Implementing VGA Application on FPGA using an Innovative Algorithm with the help of NIOS-II," International Journal of Computational Engineering Research IJCER | May-June 2012 | Vol. 2 | Issue No.3 |pp. 771775

[7] Punj Pokharel, Binod Bhatta, Anand D. Darji, "Optimized Drivers for PS/2 and VGA using HDL," Department of Electronics and Communication Engineering, Sardar Vallabbhhai National Institute of Technology, Surat, IEEE International Conference on Computer Science and Automation Engineering (CSAE), Vol:4| June, 2011

[8] A VGA Controller by Eduardo Sanchez, Ecole Polytechnique Fédérale de Laussane

[9] Xilinx. Spartan-3E FPGA Family: Data Sheet. Technical report, August 2009.

[10] Spartan-3A/3AN FPGA Starter KitlBoard User Guide.

[11] Guohui Wang and Yong Guan, "Designing of VGA Character String Display Module Base on FPGA", IEEE Conference on Intelligent Ubiquitous Computing and Education, 2009

[12] Spartan-3A/3AN FPGA UCF Constraints File for the Starter Kit Board

[13] Kai Liu, Yuliang Yang, Yanlin Zhu "Tetris game design based on the FPGA," Dept. of Communication Engineering, School of Computer \& Communication Engineering, USTB, Beijing, China, 2nd International Conference on Consumer Electronics, Communications and Networks (CECNet), April 2012.

[14] Ramneet Kaur and Balwinder Singh ,'Design and Implementation of Car Parking System on FPGA," Academic and Consultancy Services-Division, Centre for Development of Advanced

Computing(C-DAC), Mohali, International Journal of VLSI design \& Communication Systems (VLSICS) Vol.4, No.3, June 2013

[15] Circuit Design with VHDL by Volnei A. Pedroni, MIT

[16] FPGA Prototyping by VHDL examples by Pong P. $\mathrm{Chu}$ 